\title{
Structural, Mechanical, Electronic, and Magnetic Properties of LiO in Cesium Chloride, Rocksalt and Zinc-blende Structures
}

\author{
"IYORZOR, BE; BABALOLA, IM
}

\author{
Department of Physics, Faculty of Physical Sciences, University of Benin, Benin City, Nigeria \\ *Corresponding Author Email: beniyorzor@uniben.edu; Tel.: +234-0703-540-0919; michael.babalola@uniben.edu
}

\begin{abstract}
The structural, mechanical, electronic, lattice dynamic and magnetic properties of LiO in the Cesium Chloride ( $\mathrm{CsCl}$ ), Rocksalt (Rs) and Zinc-blende ( $\mathrm{ZB})$ structures is presented. The firstprinciples calculations was implemented using the spin-polarized (DFT) approach. It was observed that LiO compound exhibits ferromagnetic (FM) character at the equilibrium lattice constants in the three phases. Applying the stability conditions for cubic phases, the calculations reveal that the compound is mechanically unstable. The study also confirmed that except for the ZB structure which exhibits the brittle property, the others portray ductile nature and they are of good plasticity. We find also that the LiO structures can be synthetized, and their half-metallic property can be preserved over a broad ambit of the lattice contraction which offers the possibility to epitaxially grow such compound on a wide range of semiconductors.
\end{abstract}

\section{DOI: https://dx.doi.org/10.4314/jasem.v23i1.23}

Copyright: Copyright $\odot 2019$ Iyorzor and Babalola. This is an open access article distributed under the Creative Commons Attribution License (CCL), which permits unrestricted use, distribution, and reproduction in any medium, provided the original work is properly cited.

Dates: Received: 31 December 2018; Revised: 14 January 2019; Accepted 29 January 2019

Keywords: Half-metallic ferromagnetism; Spin-polarization; Band structure; Density of state

Half-metallic ferromagnetism (HMF) has been the center of attraction in the recent past. The dual character which the (HMF) exhibits, either as a metal in one spin channel or as a semiconductor (or insulator) in the other spin channel within the Fermi level make them very useful in spintronics device applications. de Groot at al., (1983) first predicted HMF in Heusler alloys in a calculation using the augmented-spherical-wave method. Since then, there have been several HMF theoretical predictions and confirmed experimentally, such as $\mathrm{Zn}_{1-x} \mathrm{Cr}_{x} \mathrm{Se}$ (Xin-feng and Yuan-min, 2009), XY (X = Cr, V, Mn; $\mathrm{Y}=\mathrm{P}, \mathrm{N}, \mathrm{Sb}, \mathrm{As}, \mathrm{S}, \mathrm{Te}, \mathrm{Se}$ ) (Galanakis and Mavropoulos, 2003), $C d_{1-x} C r_{x} T e$ Noor et al., (2011), and $A l_{1-x} C r_{x} A s$ (Zhao et al., 2009). Recently, in the zinc-blende (ZB) structure, the electronic, structural and magnetic properties of $C d_{1-x} V_{x} S e$ were investigated usig the First Principles Calculations (Ahmadian and Makaremi, 2012). The results confirmed that it has half-metallic property and has a total magnetic moment of $3 \mu B$. Also, in Gao et al., (2007), the ZB structure, the electronic and magnetic properties of $B C(B=$ $\mathrm{Mg}, \mathrm{Ca}, \mathrm{Sr}, \mathrm{Ba})$ compounds were calculated, the (HMF) character was confirmed, with a large halfmetallic band gaps up to $0.8 \mathrm{ev}$ and their halfmetallic properties were preserved for lattice constants compression 14\%,13\%, and $9 \%$ for $\mathrm{CaC}, \mathrm{SrC}$ and $\mathrm{BaC}$ respectively. In the works of Zhang et al., (2003), HMF was also predicted in ZB type $C r B i$ with a magnetic moment of $3 \mu B$ per formula and its half-metallicity was preserved over a wide range of the lattice constant (between -8 and $8 \%$ ). And based on First -Principles calculation, Akinaga et al., (2000), predicted a (HMF) in ZB-type $\mathrm{CrAs}$ and also fabricated film on substrate of $\mathrm{GaAs}$ compound. Recently, Lei et al., (2016) reported the presence of HMF in Rs $X O(X=L i, K, R b, C s)$. They studied the structural, electronic and magnetic properties of these compound only in the Rs structure. In the present paper, we extended the earlier report on $\mathrm{Rs} \mathrm{LiO}$ compound to other structures such as Cesium Chloride ( $\mathrm{CsCl}$ ), Rocksalt (Rs) and Zinc-blende (ZB) structures.

\section{MATERIALS AND METHODS}

We performed the First-Principles total energy calculations with a basis set of a Plane-AugmentedWave Pseudopotential (PAW PP) method using the QE code as carried out in Giannozzi et al. (2009). The generalized gradient approximation (GGA) was equally implemented as in Perdew et al., (1996). To achieve convergence, a PAW basis set with kinetic energy cut-off of $65 R y$, charge density cut-off $260 R y$, 
convergence threshold of $1.0 \times 10^{-6}$ and lattice parameters of $2.533 \AA, 4.121 \AA$ and $4.474 \AA$ in $\mathrm{CsCl}$, Rs and ZB structures were applied respectively. To obtain the ground state properties of the $\mathrm{LiO}$ compound in these structures, structurally the lattice parameter was optimized through energy minimization as shown in Fig. 1. Fitting the data generated from energy-lattice parameters to the Birch-Murnaghan equation of state, the equilibrium lattice constant and bulk modulus were obtained.
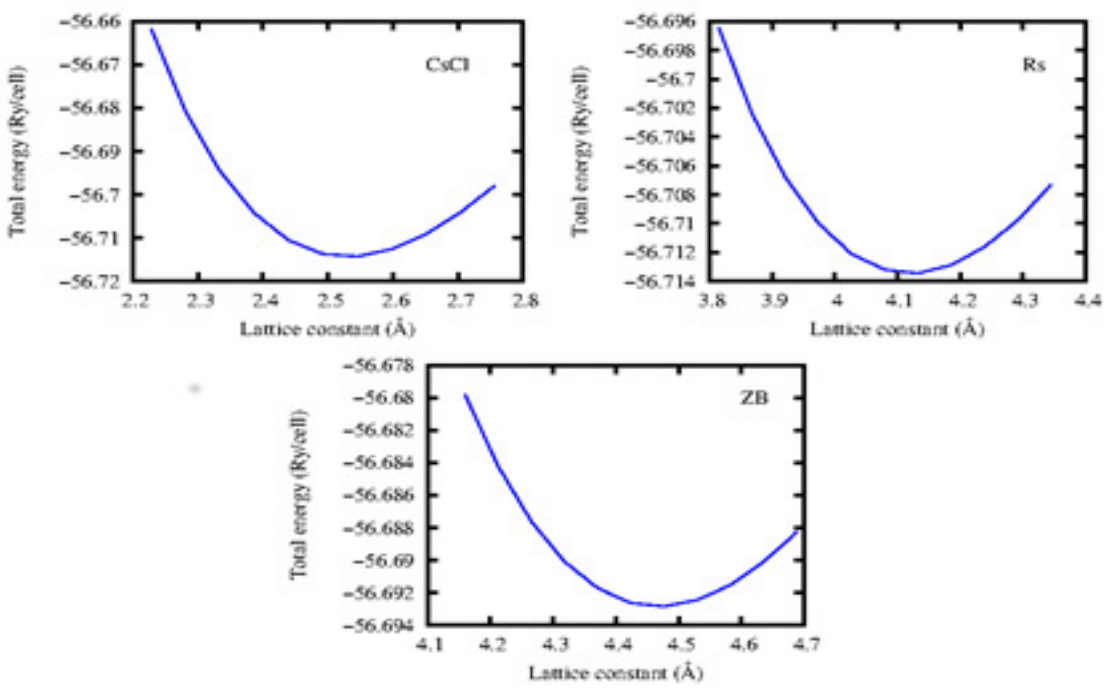

Fig 1. The total energies per unit cell as a function of lattice constants for the $\mathrm{CsCl}$, Rs and $\mathrm{ZB}$ structures of $\mathrm{LiO}$.

\section{RESULTS AND DISCUSSIONS}

Structural and Mechanical Properties: In the $\mathrm{CsCl}$, Rs and ZB structures, the total energy as a function of the lattice constant of the $\mathrm{LiO}$ compound was investigated by carrying out a spin polarized and unpolarized calculations. There was a fair agreement between the present results and the other available theoretical results as summarized in Table 1 . Both the spin-polarized ferromagnetic phase (FM) and the spin-unpolarized non-ferromagnetic phase (NM) where studied. It was observed that the ferromagnetic magnetic states are more stable than the nonferromagnetic states in the three structures investigated. These results confirm that the $\mathrm{LiO}$ compound exhibits ferromagnetic character as reported in Fig. 2. The formation energy $\Delta \mathrm{H}$ of the $\mathrm{LiO}$ in the three structures were investigated to verify whether they can be synthetized experimentally. The calculation was done by using equation 1 :

$$
\Delta H=E_{\text {total }}^{\text {comp }}-\left(E_{\text {bulk }}^{L i}+E_{\text {bulk }}^{O}\right)(1)
$$

Where $E_{\text {bulk }}^{L i}$ and $E_{\text {bulk }}^{O}=$ the total energy of Li and $O$ respectively, and $E_{\text {total }}^{\text {comp }}=$ the total energy of the LiO compound.

The calculated values of the formation energy for the three structures are presented in Table 1. From the results, it is obvious that this compound can be synthetized because of the negative formation energy that were obtained. To measure the strength of the binding force of the atoms in the compound, we investigated the cohesion energy $\Delta \mathrm{C}$ using equation 2:

$$
\Delta C=E_{\text {cohesion }}^{\text {comp }}=E_{\text {atom }}^{L i}+E_{\text {atom }}^{O}-E_{\text {total }}^{\text {comp }}(2)
$$

Where $E_{\text {atom }}^{L i}$ and $E_{\text {atom }}^{O}=$ the total energy of $\mathrm{Li}$ and $\mathrm{O}$ atoms respectively, and $E_{\text {total }}^{\text {comp }}=$ the total energy of the $\mathrm{LiO}$ compound.

The positive values obtained from the calculation indicate that it is stable in the three structures investigated. From the results of the $\Delta \mathrm{H}$ and $\Delta \mathrm{C}$ one can infer the possibility of its existence in real life. The mechanical properties of $\mathrm{LiO}$ in the $\mathrm{CsCl}$, $\mathrm{Rs}$ and ZB structures have been computed and presented in Table 2. It is essential to note that the results of the elastic constants is a function of the stability and stiffness of the materials (Wu et al., 2007). Therefore, applying these conditions for cubic phases as presented in Sin'ko and Smirnow (2002), we found that $\mathrm{LiO}$ is not mechanically stable in the structures studied since some of the conditions were not satisfied. 
Table 1. The Structural properties: the lattice constant a ( $\AA$ ) , bulk modulus B ( GPa ), formation energy $\Delta H(\mathrm{eV})$, cohesion energy $\Delta C$ ( $\mathrm{eV}$ ), half-metallic gap $\mathrm{E}_{\mathrm{HM}}(\mathrm{eV})$, energy band gap $\mathrm{E}_{\mathrm{g}}(\mathrm{eV})$ and other theoretical results (T.R.) for the various sructures.

\begin{tabular}{lllllll}
\hline Structure & $\mathbf{a}$ & $\mathbf{B}$ & $\Delta H$ & $\Delta C$ & $E_{H M}$ & $E_{g}$ \\
\hline $\mathrm{CsCl}$ & 2.533 & 79.22 & -3.72 & 4.91 & 0.63 & 8.05 \\
$\mathrm{Rs}$ & 4.121 & 65.25 & -3.71 & 4.90 & 0.94 & 8.28 \\
Other T.R. & $4.11^{\mathrm{a}}$ & -- & $-4.21^{\mathrm{a}}$ & $5.49^{\mathrm{a}}$ & -- & -- \\
$\mathrm{ZB}$ & 4.474 & 47.17 & -3.43 & 4.62 & 0.78 & 5.89 \\
\hline \multicolumn{7}{c}{$\mathrm{a}=$ Ref. (Lei et al., 2016) }
\end{tabular}
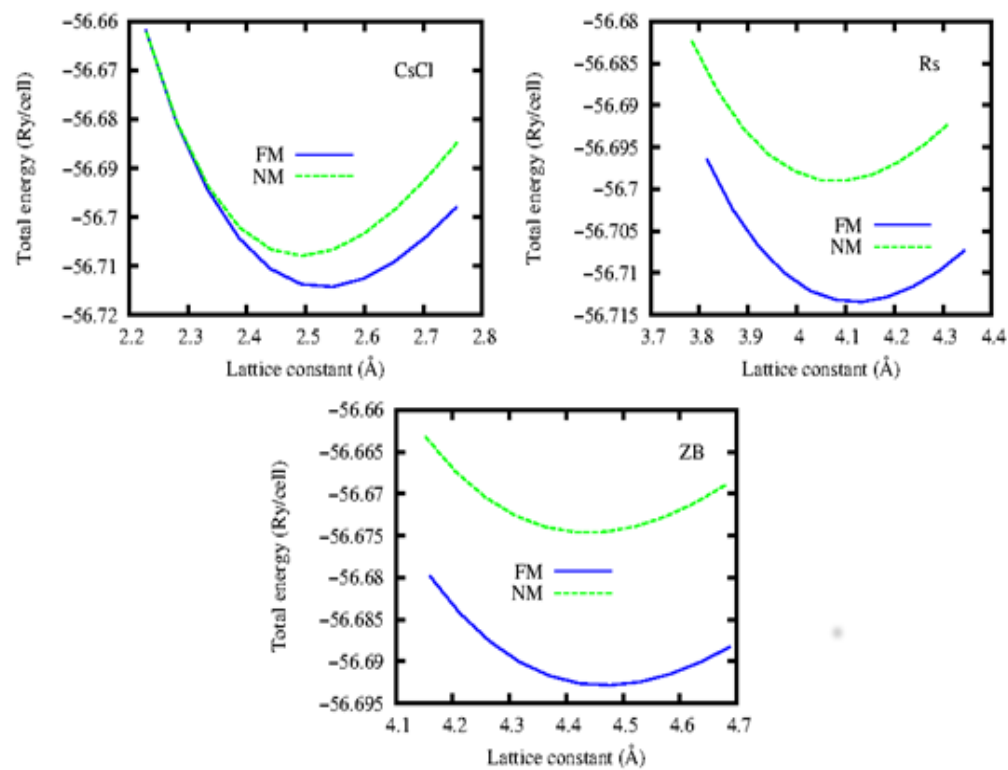

Fig 2. The total energies per unit cell as a function of lattice constants for the ferromagnetic (FM) and non-magnetic (NM) states of LiO in $\mathrm{CsCl}$, Rs and ZB structures.

The bulk (B), Young (E), and shear (G) moduli are also parameters used to quantify the mechanical properties of solids. They show the extent of resistance of the alloys or compounds to volume and shear deformation respectively.

Table 2. The mechanical properties: the elastic constants $C_{11}, C_{12}$ and $C_{44}(\mathrm{GPa})$, Zener anisotropy $A$, shear modulus $G(\mathrm{GPa})$, Young modulus $E(\mathrm{GPa})$, Poisson's ratio $v$, hardness $H(\mathrm{GPa})$, and the $B / G$ ratio for the various structures of $\mathrm{LiO}$.

\begin{tabular}{llll}
\hline Compound & CsC1 & Rs & ZB \\
$C_{H}$ & 207.24 & 118.05 & 20.61 \\
$C_{H}$ & 15.21 & 38.84 & 60.45 \\
$C_{A 4}$ & -4.41 & -12.41 & -39.16 \\
$A$ & -0.0417 & -0.3133 & 1.9654 \\
$G$ & 35.79 & 8.39 & -31.46 \\
$E$ & 93.24 & 24.14 & -121.38 \\
$\pi$ & 0.3038 & 0.4383 & 0.9289 \\
$H$ & -4.677 & -0.3452 & 8.9965 \\
$B / G$ & 2.213 & 7.777 & -1.499 \\
\hline
\end{tabular}

The higher their values, the stronger the deformation resistance offered by the solid. From Tables 1 and 2, it is obvious that the deformation resistance offered by the compound decreases in trend in three structures investigated from $\mathrm{CsCl} \rightarrow \mathrm{Rs} \rightarrow \mathrm{ZB}$.
An empirical relation to determine the plastic properties of materials is given by the ratio of the bulk to the shear moduli $(\mathrm{B} / \mathrm{G})$. The critical threshold value of the ratio for delineating ductile from brittle materials is about 1.75 (Pugh, 1954). From the present result in Table 2, it shows that except for the ZB structure which portrays a brittle character, the other structures studied exhibit ductile nature since the values exceed the critical threshold value of the $\mathrm{B} / \mathrm{G}$ ratio. The anisotropy factor $\mathrm{A}$ of the $\mathrm{LiO}$ was also investigated. The Zener anisotropy factor $=1$ for isotropic materials, otherwise it is anisotropic. The present result shows that the structures are isotropic. The Poisson's ratio reveals the nature of the bonding forces in solids and it is also used to predict the stability of the material against shear stress. The value range is the order $0<v<0.5$ (Boucetta, 2014). The value of Poisson's ratio indicates a large volume compression of the material. The higher the Poisson's ratios, the better the plasticity. From present results in Table 2, $\mathrm{LiO}$ compounds are of good plasticity. Microhardness $\mathrm{H}$, a mechanical property that measures the resistance to a localized plastic deformation of a material was studied, and it 
is given by the relation, Cheng, 2013; Yousef,ElAdawy and El-KheshKhany, (2006)

$$
H=\frac{(1-2 v)}{6(1+v)}(3)
$$

Comparing the present results for $H$ in Table 2 with the Mohs scale, ranging from 1 on the soft end for talc to 10 for diamond (Callister, 2006), it was observed that the $\mathrm{Rs} \mathrm{CsCl}$ structures are extremely soft material. While the ZB appears harder.

Electronic Properties: We present the calculated spin-polarized band structures of $\mathrm{LiO}$ compound in the $\mathrm{CsCl}$, Rs and $\mathrm{ZB}$ structures in Figs. 3 - 5. As predicted, the minority-spin channel is metallic whereas in the majority-spin channel it portrays a semiconductor property since it has an energy band gap situated around the Fermi level. In Fig. 6, we present the spin-resolved (PDOS) of $\mathrm{LiO}$ at its equilibrium lattice constants in the three structures. From the three structures, the plots revealed that the major contribution that lead to the half-metallic character came from the $\mathrm{O}-2 \mathrm{p}$ orbital at the Fermi level. In the valence bands, it was found that the $\mathrm{O}$ atoms played dominant role while in the conduction bands, the $\mathrm{Li}$ atoms widely dominated the band especially the $\mathrm{Li}-2 \mathrm{~s}$ orbital as observed in the three structures.
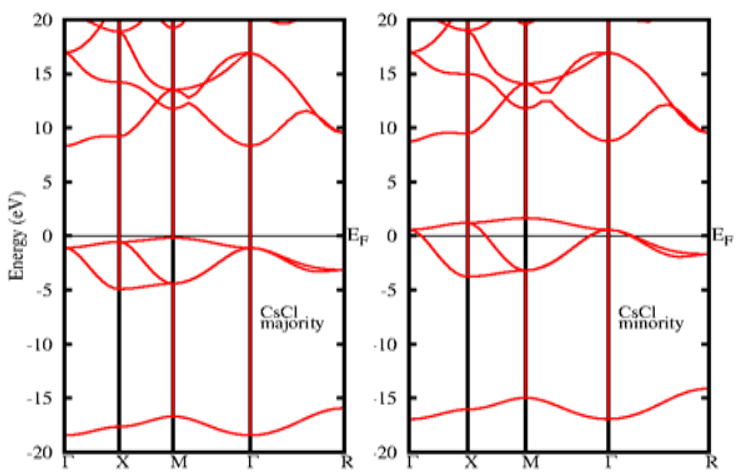

Fig 3. Electronic band structures of $\mathrm{LiO}$ in $\mathrm{CsCl}$ structure.

It was also observed from the band structures that they are direct band gap HM ferromagnets and semiconductors. The values of the HM band gaps and the energy band gaps are presented in Table 1. From the PDOS graphs, it can be seen that similar hybridizations between the $\mathrm{Li}$ and $\mathrm{O}$ orbitals occurred in the three structures. At the lower end of the valence bands, there exist a weak hybridization between the $\mathrm{Li}-2 \mathrm{p}$ and $\mathrm{O}-1$ s orbitals. At the Fermi level, the hybridization between the $\mathrm{Li}-1 \mathrm{~s}, \mathrm{Li}-2 \mathrm{~s}$ and $\mathrm{O}-2 \mathrm{p}$ is equally very weak. And at the conduction bands we observed stronger hybridization between $\mathrm{Li}-2 \mathrm{p}$ and $\mathrm{O}-1$ s orbitals. This features indicate that the covalent bond between these two atoms is weak, and could be responsible for the mechanical instability observed in the calculation.
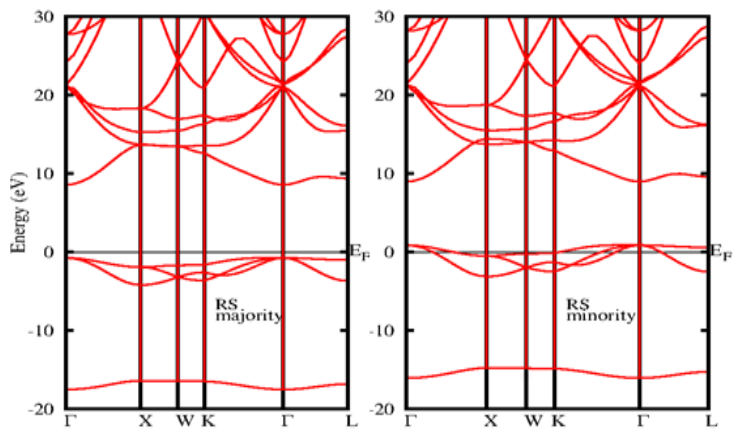

Fig 4. Electronic band structures of $\mathrm{LiO}$ in Rs structure.
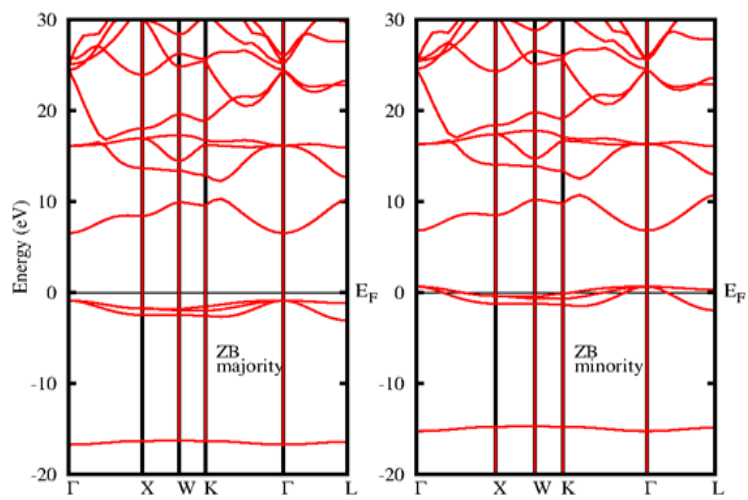

Fig 5. Electronic band structures of $\mathrm{LiO}$ in $\mathrm{ZB}$ structure.

Magnetic Properties: The magnetism in these three structures was investigated and presented in Table 3 and Fig. 7.

Table 3. The magnetic properties: the total magnetic moment $M_{\text {tot }}$ $(\mu B)$, atomic magnetic moments of $\mathrm{Li}$ and $\mathrm{O} ; M_{L i}(\mu B)$ and $M_{O}(\mu B)$ , and magnetic ground states $M g$ for the various sructures

\begin{tabular}{lllll}
\hline Structure & $M_{\text {tot }}$ & $M_{L i}$ & $M_{O}$ & $M g$ \\
\hline $\mathrm{CsCl}$ & 1.00 & -0.0227 & 1.0577 & $\mathrm{FM}$ \\
$\mathrm{Rs}$ & 1.00 & -0.0208 & 1.0333 & $\mathrm{FM}$ \\
Other T.R. & $1.00^{\mathrm{a}}$ & -- & - & $\mathrm{FM}$ \\
$\mathrm{ZB}$ & 1.00 & -0.0222 & 1.0321 & $\mathrm{FM}$ \\
\hline \multicolumn{5}{l}{$\mathrm{a}=$ Ref. (Lei et al., 2016) }
\end{tabular}

The local magnetic moments are approximately similar. Also, it was observed that the value of the magnetic moment is preserved until a critical value of the strained lattice constants of $2.32 \AA, 3.38 \AA$ and $2.36 \AA$ for $\mathrm{CsCl}$, Rs and $\mathrm{ZB}$ respectively. From Fig. 6, it is clear that the total magnetic moment of $\mathrm{LiO}$ mainly originate from $\mathrm{O}-2 \mathrm{p}$ orbitals of the $\mathrm{O}$ atom and with little contribution from the $\mathrm{Li}-2$ s orbital of the $\mathrm{Li}$ atom. 

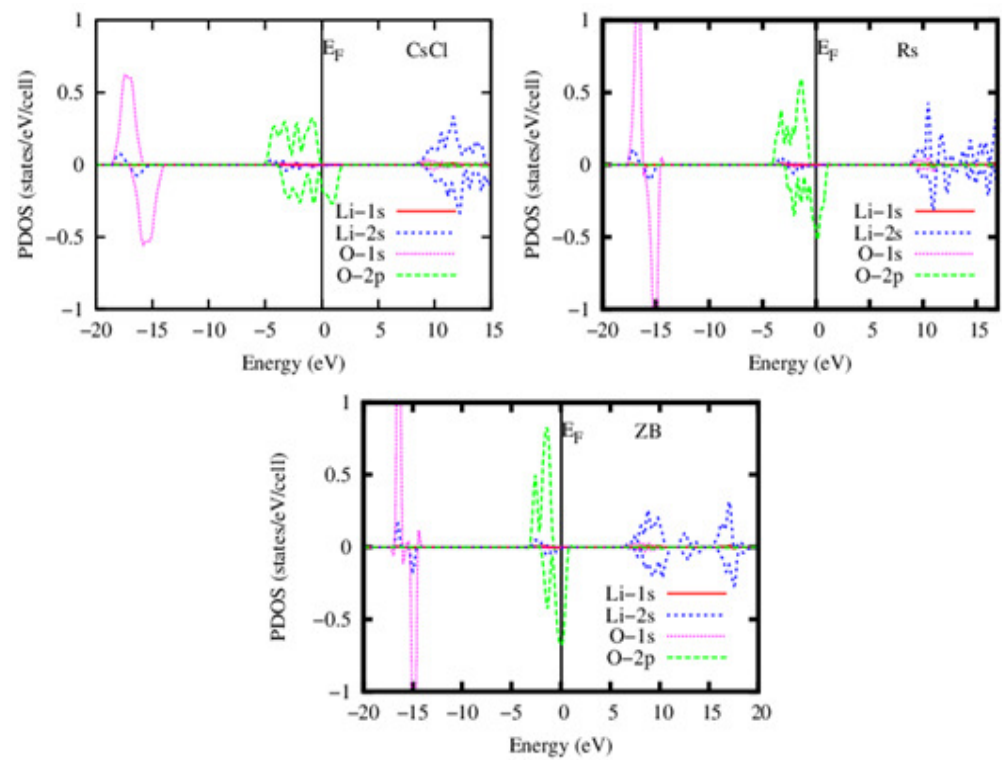

Fig 6. The spin-dependent partial density of states of $\mathrm{LiO}$ in the three structures, with positive and negative DOS corresponding to the majority and minority states.

The other property studied in this work is phonon dispersion curves. Among the three structures, only the $\mathrm{CsCl}$ is found to be dynamically stable as presented in Fig. 8

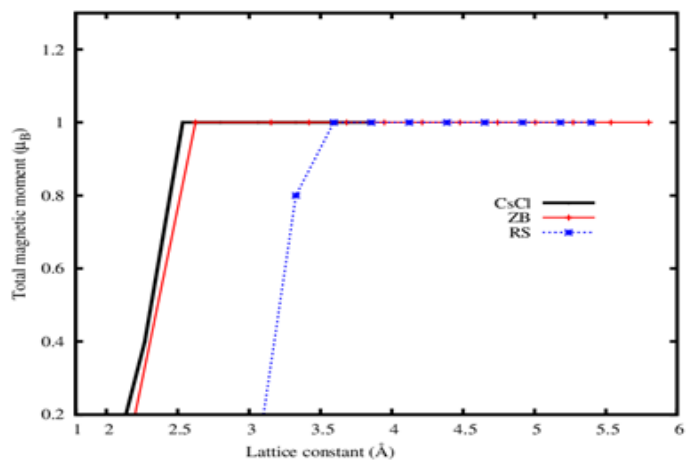

Fig 7. Total magnetic moment per formular unit as a function of lattice constants for $\mathrm{LiO}$ in the three structures.

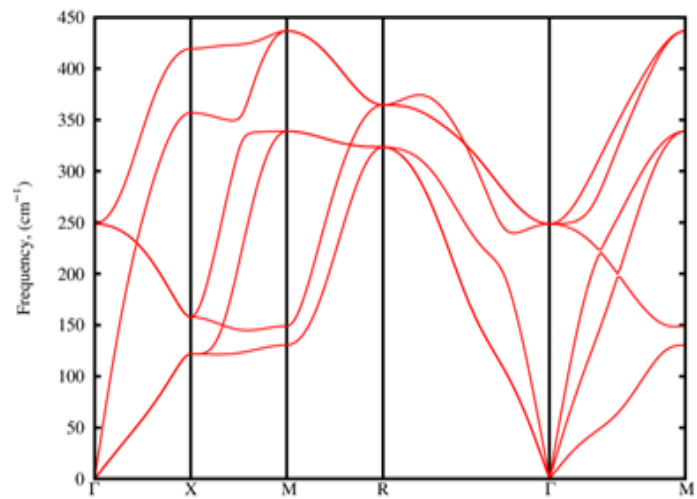

Fig 8. Phonon dispersion curves along symmetry directions of the $\mathrm{LiO}$ in the $\mathrm{CsCl}$ structure.
Conclusions: From the investigation of the spinpolarized (FM) and spin-unpolarized (NM) phases, it was found that (FM) states are more stable than (NM) states, hence the $\mathrm{LiO}$ compound exhibits (FM) character. In the calculation of the resistance to volume and shear deformation, it was found that $\mathrm{CsCl}$ structure offered highest deformation resistance compared to others. Furthermore, it was observed that their half-metallic property can be preserved up to contraction of the lattice constant of $8 \%, 18 \%$, and $47 \%$ for $\mathrm{CsCl}, \mathrm{Rs}$ and $\mathrm{ZB}$ respectively, which provides the chances of epitaxially grow $\mathrm{LiO}$ on a broad ambit of semiconductors.

Acknowledgement:We wish to thank the (ICTP) for reference articles used in this study.

\section{REFERENCES}

Ahmadian F. and Makaremi N. First principles study of half-metallic ferromagnetism in zincblende $C d_{1-x} V_{x}$ Se. Solid State Communications 2012, 152 (17), 1660-1665.

Akinaga H, Manago T. and Shirai M. Material Design of Half-Metallic Zinc-Blende CrAs and the Synthesis by Molecular-Beam Epitaxy. Jpn. J. Appl. Phys. 2000, 39, Pt2, No.11B, L1118-L1120.

Boucetta S. Theoretical study of elastic, mechanical and thermodynamic properties of $\mathrm{MgRh}$ intermetallic compound. Journal of Magnesium and Alloy 2014, 2 , 59-63. 
Callister W. D.; Mechanical Properties of Metals. In Material Science and Engineering. An Introduction. Editor, Kenneth Santor; John Wiley and Sons, Inc.: New York, U.S.A., 2006; pp. 155-157.

Cheng, H. C., Yu, C. F., and Chen, W. H. Firstprinciples density functional calculation of mechanical, thermodynamic and electronic properties of CuIn and $\mathrm{Cu} 2$ In crystals. Journal of Alloys and Compounds 2013, 546, 286-295.

de Groot R., Mueller F., Van Engen P. and Buschow. New class of materials: Half-metallic ferromagnets. Phys. Rev. Lett. 1983, 50 (25), 2024-2027.

Galanakis, I. and Mavropoulos, P. Zinc-blende compounds of transition elements with $\mathrm{N}, \mathrm{P}, \mathrm{As}$, $\mathrm{Sb}, \mathrm{S}, \mathrm{Se}$, and Te as half-metallic systems. Phys. Rev. B. 2003, 67(10), 104417-104424.

Gao G. Y., Yao K. L., Sasioglu E., Sandratskii L. M. and Jiang J. L. Half-metallic ferromagnetism in zinc-blende $\mathrm{CaC}, \mathrm{SrC}$, and $\mathrm{BaC}$ from first principles. Phys. Rev. B 2007, 75, 1744421744448.

Giannozzi, P., Baroni, S., Bonini, N., Calandra, M., Car, R., Cavazzoni, C., Ceresoli, D., Chiarotti, G.L., Cococcioni, M., Dabo, I. and Dal Corso, A. QUANTUM ESPRESSO: a modular and opensource software project for quantum simulations of materials. Journal of physics: Condensed matter. 2009, 21 (39), 395502-395538.

Lei G., Liu X., Xie H., Li L., Gao Q. and Deng J. First-principle study of half-metallic ferromagnetism in rocksalt $\mathrm{XO}(\mathrm{X}=\mathrm{Li}, \mathrm{K}, \mathrm{Rb}$, Cs). J. Magnetism and Magnetic Materials. 2016, 397, 176-180.

Noor, N. A., Ali, S., and Shaukat, A. First principles study of half-metallic ferromagnetism in Crdoped CdTe. J. Physics and Chemistry of Solids 2011, $72(6), 836-841$.
Perdew, J. P., Burke, K., and Ernzerhof, M. Generalized Gradient Approximation Made Simple. Phys. Rev. Lett. 1996, 77(18), 38653868.

Pugh S. F. The title of the cited article. The London, Edinburgh, and Dublin Philosphical Magazine and journal of science 1954, 45 (367), 823-843.

Sin'ko G. V. and Smirnow N. A. Ab initio calculations of elastic constants and thermodynamic properties of bcc, fcc, and hcp Al crystals under pressure. J. Phys. Condens. Matter 2002, 14, 6989-7005.

Wu, Z., Hao, X., Liu, X. and Meng, J. Structures and elastic properties of OsN 2 investigated via Firstprinciples density functional calculations. Phys. Rev. B. 2007, 75 (5), 054115-054120.

Xin-feng Ge and Yuan-min Zhang. First-principles study of half-metallic ferromagnetism in $\mathrm{Zn} 1$ $\mathrm{x} \mathrm{Cr} \times \mathrm{Se} . J$. Magnetism and Magnetic Materials. 2009, 321, 198-202.

Yousef, El-Adawy, A., and El-KheshKhany, N. Effect of rare earth $\left(\mathrm{Pr}_{2} \mathrm{O}_{3}, \mathrm{Nd}_{2} \mathrm{O}_{3}, \mathrm{Sm}_{2} \mathrm{O}_{3}, \mathrm{Eu}_{2} \mathrm{O}_{3}\right.$ , $\mathrm{Gd}_{2} \mathrm{O}_{3}$ and $\mathrm{Er}_{2} \mathrm{O}_{3}$ ) on the acoustic properties of glass belonging to bismuth-borate system. Solid state communications 2006, 139(3), 108113.

Zhang, M., Hu, H., Liu, G., Cui, Y., Liu, Z., Wang, J., Wu, G., Zhang, X., Yan, L., Liu, H. and Meng, F. Half-metallic ferromagnetism in zinc-blende $\mathrm{CrBi}$ and the stability of the half-metallicity of zinc-blende $\operatorname{CrM}(M=P, A s, S b, B i) . \quad J$. Physics: Condensed Matter 2003, 15(29), 50175024.

Zhao, Y. H., Zhao, G. P., Liu, Y., and Liu, B. G. Structural stability and half-metallicity of the zinc-blende phase of $A l_{1-x} C r_{x} A S$ : Densityfunctional study. Phys. Rev. B 2009, 80, 224417. 